\title{
Obtenção de gasolina verde por destilação fracionada de produtos líquidos orgânicos oriundos do craqueamento térmico-catalítico usando diferentes porcentagens de catalisador
}

Obtaining of green gasoline by fractional distillation of organic liquid products from the thermal-catalytic cracking using different percentages of catalyst

\author{
A. A. Mancio ${ }^{1 *}$; S. A. P da Mota ${ }^{2}$;. E. P. Borges ${ }^{3} ;$ N. T. Machado ${ }^{4}$ \\ ${ }^{1}$ Programa de Pós-Graduação em Engenharia de Recursos Naturais da Amazônia/Thermtek/Faculdade de \\ Engenharia Química, Universidade Federal do Pará, 66075-110, Belém-PA, Brasil \\ ${ }^{2}$ Faculdade de Engenharia de Materiais, Universidade do Sul e Sudeste do Pará, 68505-080, Marabá-PA, Brasil \\ ${ }^{3}$ Departamento de Engenharia Química, Instituto Militar de Engenharia-IME, 22290-270, Rio de Janeiro-RJ, Brasil \\ ${ }^{4}$ Faculdade de Engenharia Química, Universidade Federal do Pará, 66075-110, Belém-PA, Brasil \\ *dedeiaam@yahoo.com.br
}

(Recebido em 04 de setembro de 2016; aceito em 17 de outubro de 2016)

\begin{abstract}
Neste trabalho, avaliou-se o efeito da destilação fracionada à pressão atmosférica de produtos líquidos orgânicos (PLOs) provenientes do craqueamento térmico-catalítico de óleo de palma bruto usando diferentes porcentagens de catalisador sobre o rendimento e a qualidade de frações de gasolina verde. Os resultados mostraram que os valores de propriedades físico-químicas das frações de gasolina verde são inferiores àqueles dos seus respectivos PLOs, indicando que a destilação fracionada melhora as propriedades físico-químicas das frações destiladas. $\mathrm{O}$ incremento na porcentagem de catalisador no craqueamento térmico-catalítico para produzir os PLOs: (1) promoveu um aumento de 1,97 a 13, $82 \% \mathrm{em}$ massa no rendimento das frações de gasolina verde; (2) as propriedades físico-químicas como densidade, viscosidade e índice de refração das frações de gasolina verde aumentaram, enquanto os valores de índice de acidez e índice de saponificação diminuíram; (3) o teor de hidrocarbonetos das frações de gasolina verde aumentou, enquanto o teor de compostos oxigenados diminuiu; (4) influenciou significativamente a distribuição do número de carbonos dos hidrocarbonetos de cada fração, as quais consistem principalmente de hidrocarbonetos com cadeias C9-C12. A classe da olefinas é a mais representativa dentro do grupo de hidrocarbonetos, seguida dos de naftênicos, parafinas normais e aromáticos. Nos compostos oxigenados, as cetonas constituem a classe com maior teor, seguido de álcoois, aldeídos e outros compostos. Neste contexto, as frações de gasolina verde podem ser utilizadas como um substituto parcial ou total da gasolina de petróleo e/ou como uma das frações que constituem o chamado "Pool" da gasolina de petróleo.
\end{abstract}

Palavras-chave: gasolina verde, destilação fracionada, biocombustíveis

This study evaluated the effect of fractional distillation at the atmospheric pressure of organic liquid products (OLPs) from the thermal-catalytic cracking of crude palm oil using different percentages of catalyst on the yield and quality of green gasoline fractions. The results showed that the physicalchemical values of the green gasoline fractions are lower than those of the respective OLPs, indicating that the fractional distillation improves the physical-chemical properties of the distilled fractions. The increase in the percentage of catalyst in the thermal-catalytic cracking to produce the OLPs: (1) promoted an increase from 1.97 to $13.82 \%$ by mass in the yield of the green gasoline fractions; (2) the physicalchemical properties such as density, viscosity and refractive index of green gasoline fractions increased, while the acid values and saponification values decreased; (3) the hydrocarbon content of the green gasoline fractions increased, whereas the content of oxygenates decreased; (4) significantly influenced the distribution of carbon number of hydrocarbons of the green gasoline fractions, which consist mainly of hydrocarbons with chains $\mathrm{C} 9-\mathrm{C} 12$. The class of olefins is the most representative of the hydrocarbon group, then the naphthenic, normal paraffin, and aromatics. In the oxygenated compounds, ketones constitute the class with the highest content, followed by alcohols, aldehydes, and other compounds. Therefore, green gasoline fractions may be used as a partial or total replacement for petroleum gasoline or one of the fractions which constitute the so-called "pool" of the petroleum gasoline. 


\section{INTRODUÇÃO}

Uma das formas mais promissoras para a produção de combustíveis líquidos e produtos químicos a partir de biomassa triglicerídica é a pirólise, também conhecida como craqueamento. A pirólise de biomassa derivada de triglicerídeos tem sido estudada em diferentes condições reacionais, na ausência de catalisador (craqueamento térmico) e na presença de catalisador (craqueamento térmico-catalítico) para a obtenção de produtos similares aos combustíveis de origem fóssil [1].

O craqueamento térmico-catalítico de óleos (triglicerídeos) pode produzir biocombustíveis líquidos, biocombustíveis gasosos, coques e alcatrões, dependendo da seletividade do catalisador utilizado no processo. Os biocombustíveis líquidos, os quais são denominados de Produto Líquido Orgânico (PLO), produzidos a partir desse processo contêm parafinas lineares e cíclicas, olefinas, aldeídos, cetonas e ácidos carboxílicos [2].

O PLO proveniente do craqueamento de óleos vegetais, gorduras animais e resíduos constituídos por materiais de origem triglicerídica consiste em hidrocarbonetos que estão distribuídos nas faixas de temperatura de destilação de alguns derivados de petróleo como a gasolina, o querosene de aviação e o diesel. Portanto, é fundamental aplicar processos de separação como a destilação sobre os PLOs obtidos pelos mais variados sistemas de reação a fim de se ter como resultado frações destiladas com propriedades físicas e químicas melhores do que aquelas apresentadas pela alimentação (PLO) e/ou dentro dos limites especificados pelas agências regulamentadoras de cada país para os referidos combustíveis derivados do petróleo. Ao atender tais requisitos é possível utilizá-las tanto como um substituto parcial ou total do combustível de transporte líquido como matéria prima de uma biorrefinaria.

A destilação a pressão atmosférica, destilação a vácuo, destilação por arraste a vapor e alguns outros tipos de destilação têm sido aplicados ao fracionamento do PLO. Devido à sua complexa composição, a temperatura de ebulição do PLO pode iniciar com valores inferiores a $100{ }^{\circ} \mathrm{C}$, a pressão atmosférica, e, em seguida, a destilação prossegue na faixa de $250-280{ }^{\circ} \mathrm{C}$. A partir da caracterização da fração orgânica destilada, concluiu-se que a mesma apresenta uma qualidade muito melhor do que o PLO bruto, visto que a fração orgânica destilada contém pouca água, compostos oxigenados em menor quantidade e valor de aquecimento mais elevado [3].

Há inúmeras pesquisas na literatura sobre craqueamento térmico e craqueamento térmicocatalítico de biomassa triglicerídica. Porém, diante do número de estudos realizados na área, são poucos que ampliam a pesquisa para investigar a etapa de fracionamento do PLO. Dentre as poucas pesquisas na referida temática, é possível encontrar estudos de craqueamento térmico e térmico-catalítico de biomassa triglicerídica em que os experimentos foram realizados em reatores acoplados a uma unidade de destilação para que as frações líquidas fossem imediatamente separadas durante o processo de craqueamento, evitando uma posterior etapa de destilação. É o caso das pesquisas desenvolvidas por Dandik et al. (1998) [1], Schwab et al. (1988) [4] e Prado e Antoniosi Filho (2009) [5]. Em contrapartida, existem trabalhos que investigaram o fracionamento do PLO em unidades de craqueamento e destilação de forma separada, como os trabalhos desenvolvidos por Twaiq et al. (2004) [6], Ooi et al. (2004) [7], Wiggers et al. (2009) [8], Santos et al. (2010) [9], Buzetzki et al. (2011) [10], Wiggers et al. (2013) (11)] e Mota et al. (2014) [12], para citar alguns exemplos.

Schwab et al. (1988) [4] realizaram uma espécie de decomposição térmica do óleo de soja em um aparato experimental de destilação padrão da ASTM. Os resultados de GC-MS mostraram que aproximadamente $75 \%$ dos produtos consistiam de alcanos, alcenos, aromáticos e ácidos carboxílicos. O número de carbonos desses compostos variou de 4 a valores superiores a 20.

Twaiq et al. (2004) [6] realizaram o fracionamento de produto líquido em uma micro unidade de destilação a vácuo nas condições de $10 \mathrm{~Pa}$ e $200{ }^{\circ} \mathrm{C}$ por 30 minutos. Além disso, os referidos autores denominaram a fração destilada de produto líquido orgânico e o produto de fundo foi considerado como óleo residual.

Ooi et al. (2004) [7] realizaram o fracionamento de produto líquido em uma micro unidade de destilação a vácuo nas condições de $5 \mathrm{~Pa}$ e $200{ }^{\circ} \mathrm{C}$ por 30 minutos. A fração destilada foi denominada de produto líquido orgânico, o qual foi definido de acordo com a faixa de ebulição 
dos produtos de petróleo que compreende as seguintes categorias: fração de gasolina (60$\left.120^{\circ} \mathrm{C}\right)$, fração de querosene $\left(120-180{ }^{\circ} \mathrm{C}\right)$ e fração de diesel $\left(180-200{ }^{\circ} \mathrm{C}\right)$.

Wiggers et al. (2009) [8] realizaram o fracionamento do produto líquido orgânico em duas frações: gasolina (temperatura ambiente até $200{ }^{\circ} \mathrm{C}$ ) e diesel $\left(150-400^{\circ} \mathrm{C}\right)$. De acordo com os resultados, a destilação do PLO favoreceu a produção de biocombustíveis com características físico-químicas semelhantes aos combustíveis de petróleo.

Santos et al. (2010) [9] realizaram a destilação de produtos líquidos orgânicos em quatro frações nas seguintes faixas de temperatura de destilação (TD): (a) $\mathrm{TD}<80{ }^{\circ} \mathrm{C}$, (b) $80 \leq \mathrm{TD} \leq 140{ }^{\circ} \mathrm{C}$, (c) $140 \leq \mathrm{TD}<200^{\circ} \mathrm{C}$ e (d) $\mathrm{TD} \geq 200{ }^{\circ} \mathrm{C}$ (fração pesada). A fração obtida acima de $200{ }^{\circ} \mathrm{C}$ foi isolada e caracterizada por GC-FID, GC-MS e FT-IR. De acordo com os resultados obtidos, verificou-se a formação de olefinas, parafinas e alguns compostos oxigenados (ácidos carboxílicos ésteres). Além disso, as seguintes propriedades físico-químicas foram determinadas: densidade, viscosidade, curva de destilação, resíduo de carbono, corrosividade ao cobre, índice de cetano, índice de acidez e valor de aquecimento, as quais foram comparadas com as especificações brasileiras para o diesel de petróleo.

Wiggers et al. (2013) [11] produziram produto líquido orgânico através de craqueamento térmico e, em seguida, submeteram o PLO a um fracionamento por destilação simples a fim de obter produtos purificados na faixa de temperatura de destilação de nafta leve, a qual corresponde a temperatura inferior a $180^{\circ} \mathrm{C}$. Os resultados mostram que a destilação favoreceu a concentração de hidrocarbonetos (com número de carbonos igual ou superior a 14) nas fases mais pesadas.

Mota et al. (2014) [12] realizaram o fracionamento de PLO em uma coluna de destilação do tipo Vigreux com o objetivo de obter frações destiladas na faixa de temperatura de destilação do diesel de petróleo. Os autores fixaram a faixa de temperatura de destilação em $235-305^{\circ} \mathrm{C}$ e, denominaram a fração destilada de diesel verde, o qual consistiu de hidrocarbonetos que variaram de $\mathrm{C} 6$ a C21. Apesar do diesel verde apresentar ampla faixa de hidrocarbonetos em relação ao número de carbonos, constatou-se que os maiores teores correspondiam a hidrocarbonetos C12-C15.

Neste contexto, o presente trabalho tem como objetivo principal avaliar o efeito da destilação fracionada à pressão atmosférica de produtos líquidos orgânicos provenientes do craqueamento térmico-catalítico de óleo de palma bruto usando diferentes porcentagens de carbonato de sódio sobre o rendimento e a qualidade de frações de gasolina verde obtida na faixa de temperatura de destilação de $28-160{ }^{\circ} \mathrm{C}$.

\section{MATERIAL E MÉTODOS}

\subsection{Produto Líquido Orgânico (PLO)}

No presente trabalho foram utilizados quatro Produtos Líquidos Orgânicos (PLO) diferentes, $\mathrm{PLO}_{1}, \mathrm{PLO}_{2}, \mathrm{PLO}_{3}$ e $\mathrm{PLO}_{4}$, os quais foram obtidos através de craqueamento térmico-catalítico de óleo de palma bruto em uma planta piloto empregando, respectivamente, 5, 10, 15 e 20\% de carbonato de sódio como catalisador, conforme descrito por Mota et al. (2014) [12].

\subsection{Caracterização do PLO}

No trabalho desenvolvido previamente por Mancio et al. (2016) [13], os PLOs foram caracterizados através de análises físico-químicas e análise de CG-EM.

\subsection{Aparato experimental da destilação fracionada}

O aparato experimental da destilação fracionada para obter frações de gasolina verde consiste de acessórios, vidrarias e equipamentos, conforme descritos por Mota et al. (2014) [12]. Porém, enquanto Mota et al. (2014) [12] utilizaram uma coluna de destilação do tipo Vigreux 
com três estágios, no presente trabalho foi empregada uma coluna Vigreux com seis estágios visando obter frações destiladas com melhor qualidade no que tange às propriedades físicoquímicas e à composição química.

\subsection{Procedimento experimental da destilação fracionada}

A destilação fracionada em escala de bancada do PLO foi utilizada para obter frações de gasolina verde na faixa de temperatura de ebulição de $28-160{ }^{\circ} \mathrm{C}$ a pressão atmosférica.

$\mathrm{O}$ procedimento experimental iniciou com a pesagem de aproximadamente $600 \mathrm{~g}$ de PLO em uma balança semi-analítica com capacidade de $1 \mathrm{~kg}$ e precisão de 0,1 . Em paralelo, o banho ultratermostático foi programado para alcançar $10^{\circ} \mathrm{C}$ com o objetivo de condensar os gases produzidos durante a destilação. Uma vez realizada a pesagem do PLO, ele foi imediatamente introduzido no balão de fundo redondo. Em seguida, a manta de aquecimento foi ligada. $\mathrm{O}$ aquecimento foi controlado para se obter a fração de gasolina verde na faixa de temperatura preestabelecida $\left(28-160{ }^{\circ} \mathrm{C}\right)$.

As frações destiladas na faixa de temperatura preestabelecida mostraram a formação de duas fases, uma fase orgânica e uma fase aquosa, que foram separadas uma das outras ao término da destilação de cada fração. A fase orgânica foi caracterizada como a fase da leve e, a fase aquosa foi caracterizada como a fase pesada. As fases orgânicas foram denominadas de fração de gasolina verde. Após a separação das duas fases, armazenou-se a fração de gasolina verde.

Ao término da destilação fracionada, o produto de fundo foi removido do balão de fundo redondo e reservado. Como etapa final do procedimento experimental, a fração destilada, a fração aquosa e produto de fundo foram submetidos à pesagem para calcular os seus valores de rendimento, conforme a equação 1 .

$$
\text { Rendimento }(\% \text { em massa })=\frac{m_{\text {Produto desejado }}}{m_{P L O}} \times 100
$$

\subsection{Caracterização das frações destiladas}

As frações destiladas foram caracterizadas através de análises físico-químicas e análise de cromatografia gasosa acoplada à espectrometria de massas (CG-EM). A caracterização físicoquímica das frações destiladas foi realizada seguindo métodos padrões da American Oil Chemists' Society (AOCS) e da American Society for Testing and Materials (ASTM), os quais são: densidade a $20{ }^{\circ} \mathrm{C}$ (AOCS Cc 10c-95), viscosidade cinemática a $40{ }^{\circ} \mathrm{C}$ (ASTM D 445), corrosividade ao cobre (ASTM D 130), índice de acidez (ASTM D 974), índice de saponificação (AOCS Cd 3-25) e índice de refração (AOCS Cc 7-25).

A análise de CG-EM das frações de gasolina verde foi realizada utilizando um cromatógrafo gasoso acoplado a um espectrômetro de massas (Shimadzu, Modelo: GCMS-2010) e interface de QP2010. A coluna foi a RTX-5MS com comprimento de $30 \mathrm{~m}$ e diâmetro de $0,25 \mathrm{~mm}$. O hélio foi utilizado como gás transportador com uma vazão de $1 \mathrm{~mL} / \mathrm{min}$ e uma taxa de separação de 1/100. A seguinte programação de temperatura foi usada: primeiramente, elevou-se a temperatura do forno de $15^{\circ} \mathrm{C} / \mathrm{min}$ até $150{ }^{\circ} \mathrm{C}$, em seguida de $8{ }^{\circ} \mathrm{C} / \mathrm{min}$ até $200{ }^{\circ} \mathrm{C}$ e $2{ }^{\circ} \mathrm{C} / \mathrm{min}$ até $240{ }^{\circ} \mathrm{C}$. Esta temperatura foi então mantida durante $4 \mathrm{~min}$ antes de se aquecer o forno de $15^{\circ} \mathrm{C} / \mathrm{min}$ até $300{ }^{\circ} \mathrm{C}$. A temperatura do injetor e do detector foi igual a $280{ }^{\circ} \mathrm{C}$. Por último, os compostos químicos foram identificados por comparação com a biblioteca NIST05s.LIB. 


\section{RESULTADOS E DISCUSSÃO}

\subsection{Rendimento}

A Tabela 1 apresenta os resultados de rendimento dos produtos provenientes do fracionamento dos quatro PLOs utilizados no presente estudo.

Tabela 1: Rendimento dos produtos provenientes da destilação fracionada dos PLOs.

\begin{tabular}{|c|c|c|c|c|}
\hline Produtos & $\begin{array}{c}\mathrm{PLO}_{1} \\
\left(5 \% \text { de } \mathrm{Na}_{2} \mathrm{O}_{3}\right)\end{array}$ & $\begin{array}{c}\mathrm{PLO}_{2} \\
\left(10 \% \mathrm{de} \mathrm{Na}_{2} \mathrm{O}_{3}\right)\end{array}$ & $\begin{array}{c}\mathrm{PLO}_{3} \\
\left(15 \% \mathrm{de} \mathrm{Na}_{2} \mathrm{O}_{3}\right)\end{array}$ & $\begin{array}{c}\mathrm{PLO}_{4} \\
\left(20 \% \text { de } \mathrm{Na}_{2} \mathrm{O}_{3}\right)\end{array}$ \\
\hline \multicolumn{5}{|l|}{ Rendimento (\% mássica) } \\
\hline Gasolina verde $\left(28-160^{\circ} \mathrm{C}\right)$ & 1,97 & 7,09 & 11,04 & 13,82 \\
\hline Fração aquosa $\left(28-160{ }^{\circ} \mathrm{C}\right)$ & 0,58 & 0,13 & 0,75 & 0,01 \\
\hline Produto de Fundo & 97,45 & 92,78 & 88,21 & 86,17 \\
\hline Total & 100,00 & 100,00 & 100,00 & 100,00 \\
\hline
\end{tabular}

De acordo com a Tabela 1, todas as frações de gasolina verde apresentaram alguma quantidade de fração aquosa. Porém, o conteúdo de fração aquosa em todas as frações destiladas não ultrapassou 1\% em massa. Segundo Mrad et al. (2013) [14], o carbonato de sódio é um dos catalisadores que quando utilizados no craqueamento de térmico-catalítico de gordura de peixe residual produzem o menor teor de fração aquosa no produto líquido orgânico. Além disso, o trabalho realizado previamente por Mancio et al. (2016) [13] mostra que a fração aquosa é o produto do craqueamento térmico-catalítico do óleo de palma com carbonato de sódio que apresenta o menor rendimento. Além de ser o produto com o menor rendimento, a fração aquosa é separada do produto líquido orgânico antes deste último ser submetido à destilação fracionada, resultando em um baixo teor de fração aquosa nas frações de gasolina verde.

A Tabela 1 também mostra que houve um aumento gradual e significativo nos valores de rendimento das frações de gasolina verde, o qual variou de 1,97 a 13, 82,\% em massa, com o aumento do conteúdo de carbonato de sódio no craqueamento térmico-catalítico, ou seja, do $\mathrm{PLO}_{1}$ ao $\mathrm{PLO}_{4}$. Em contrapartida, verificou-se que o rendimento dos produtos de fundo diminui com o aumento do conteúdo de catalisador. Os resultados de rendimento das frações de gasolina verde obtidos no presente trabalho são similares àqueles obtidos por Li et al. (2009) [15], em que também constataram que o aumento do conteúdo de catalisador no craqueamento catalítico de óleo de algodão promove um aumento no rendimento de biogasolina.

\subsection{Caracterização das frações de gasolina verde}

A Tabela 2 apresenta os resultados referentes à caracterização físico-química das frações de gasolina verde. É importante destacar que os valores das propriedades físico-químicas apresentados na referida tabela são comparados com os limites estabelecidos pela ANP $\mathrm{N}^{\circ} 40$ (2013) [18], a qual regulamenta as especificações da gasolina automotiva no Brasil. Além disso, o fracionamento do $\mathrm{PLO}_{1}$ resultou em uma quantidade muito pequena de fração de gasolina verde, impedindo a determinação de propriedades físico-químicas como viscosidade cinemática a $40{ }^{\circ} \mathrm{C}$ e índice de acidez, conforme apresentado na Tabela 2. 
Tabela 2: Propriedades físico-químicas das frações de gasolina verde.

\begin{tabular}{|c|c|c|c|c|c|}
\hline \multirow{2}{*}{ Propriedades físico-químicas } & \multicolumn{4}{|c|}{ Fração de gasolina verde } & \multirow{2}{*}{$\begin{array}{l}\text { ANP } \\
\mathbf{N}^{\mathbf{0} 40} \\
{[18]}\end{array}$} \\
\hline & PLO $_{1}$ & $\mathrm{PLO}_{2}$ & $\mathrm{PLO}_{3}$ & $\mathrm{PLO}_{4}$ & \\
\hline Densidade a $20{ }^{\circ} \mathrm{C}\left(\mathrm{g} / \mathrm{cm}^{3}\right)$ & 0,722 & 0,739 & 0,779 & 0,788 & - \\
\hline Viscosidade cinemática a $40{ }^{\circ} \mathrm{C}\left(\mathrm{mm}^{2} / \mathrm{s}\right)$ & - & 0,74 & 0,75 & 0,79 & - \\
\hline Corrosividade ao cobre, $3 \mathrm{~h}$ a $50{ }^{\circ} \mathrm{C}$ & 1 & 1 & 1 & 1 & 1 \\
\hline Índice de acidez (mg KOH/g) & - & 7,88 & 1,28 & 0,79 & - \\
\hline Índice de saponificação (mg KOH/g) & 36,79 & 8,67 & 12,91 & 13,68 & - \\
\hline Índice de refração & 1,416 & 1,416 & 1,423 & 1,425 & - \\
\hline
\end{tabular}

Primeiramente, ao comparar os valores de propriedades físico-químicas das frações de gasolina verde apresentados na Tabela 2 com os valores dos seus respectivos PLOs, os quais estão dispostos no trabalho de Mancio et al. (2016) [13], verificou-se que a maioria daqueles valores reduziu em relação valores do PLO. Esse fato indica que a destilação fracionada melhora as propriedades físico-químicas das frações destiladas, uma vez que um dos fatores pelos quais os PLOs não podem ser aplicados diretamente como biocombustíveis é o elevado valor de propriedades como viscosidade cinemática e índice de acidez.

Em segundo lugar, verificou-se que à medida que há um aumento no conteúdo de catalisador no craqueamento térmico-catalítico para produzir o $\mathrm{PLO}_{1}, \mathrm{PLO}_{2}, \mathrm{PLO}_{3}$ e $\mathrm{PLO}_{4}$, as propriedades físico-químicas como densidade, viscosidade e índice de refração das respectivas frações de gasolina verde também aumentam. Em contrapartida, o incremento de catalisador no craqueamento para produzir os mesmos PLOs promove a redução nos valores de índice de acidez e índice de saponificação das frações de gasolina verde.

De acordo com Riazi (2005) [16] a gasolina derivada do petróleo é constituída por cadeias de hidrocarbonetos que variam de C4 a C10. As frações de gasolina verde também são constituídas por cadeias de hidrocarbonetos de diferentes tamanhos ou números de carbonos. Portanto, o aumento nos valores de densidade, viscosidade e índice de refração indicam que hidrocarbonetos com cadeias maiores, porém dentro da faixa de hidrocarbonetos da gasolina, são incorporados nas frações de gasolina verde à medida que ocorre a destilação fracionada do $\mathrm{PLO}_{1}$ ao $\mathrm{PLO}_{4}$. Esses resultados podem ser corroborados através da análise de CG-EM, em especial a Figura 2, onde se verificou que o aumento do carbonato de sódio promove um aumento na concentração de hidrocarbonetos de cadeias maiores presentes nas frações de gasolina verde.

Oasmaa et al. (2010) [17] relatam que acidez do produto líquido orgânico reflete a acidez dos seus constituintes, incluindo álcoois, cetonas, aldeídos, fenóis e, principalmente, ácidos carboxílicos. Portanto, os valores decrescentes de índice de acidez das frações de gasolina em função do teor de carbonato de sódio empregado no craqueamento térmico-catalítico são uma consequência direta da composição das frações de gasolina verde, conforme pode ser corroborado na análise de CG-EM.

Apesar de tentarmos fazer uma comparação dos resultados obtidos com aqueles estabelecidos com a ANP No40 (2013) [18] para a gasolina automotiva, é possível verificar através da Tabela 2 que dentre as propriedades físico-químicas determinadas no presente trabalho a referida agência de regulamentação estabelece limites apenas para a propriedade corrosividade ao cobre. Os resultados mostram que todas as frações de gasolina verde atenderam os limites estabelecidos para a corrosividade ao cobre.

Neste contexto, a destilação fracionada a pressão atmosférica promoveu uma melhora na qualidade das frações de gasolina verde quando comparadas com a matéria prima (PLO). De acordo com Parkash (2003) [19], a gasolina é uma mistura de frações de petróleo proveniente de uma variedade de processos de refinaria, formando um "Pool" ou um conjunto de frações que fazem parte do referido derivado de petróleo. Portanto, as frações de gasolina verde podem ser utilizadas como um substituto parcial ou total da gasolina de petróleo e/ou como uma das frações que constituem o chamado "Pool" da gasolina de petróleo. 
Os resultados da análise por CG-EM das frações de gasolina verde obtidas a partir da destilação fracionada em escala de bancada do $\mathrm{PLO}_{1}, \mathrm{PLO}_{2}, \mathrm{PLO}_{3}$ e $\mathrm{PLO}_{4}$ são apresentados na forma de cromatogramas, conforme a Figura 1. Os perfis dos quatro cromatogramas são muito semelhantes, o que indica que os compostos têm tempos de retenção semelhantes e, em geral, o que os distinguem são apenas os seus teores. De acordo com a Figura 1, os principais picos das frações de gasolina verde estão localizados no intervalo entre 4,5 e 7,5 minutos, indicando a presença de hidrocarbonetos com cadeias curtas.

A Tabela 3 fornece a lista dos compostos químicos e suas respectivas áreas dos picos identificados por análise de CG-EM para as frações de gasolina verde proveniente do fracionamento de cada PLO. Os compostos químicos presentes nas frações de gasolina verde foram classificados em dois grupos principais: hidrocarbonetos (parafinas normais, olefinas, aromáticos e naftênicos) e compostos oxigenados (ácidos carboxílicos, álcoois, aldeídos, cetonas e outros), os quais estão dispostos na Tabela 4.

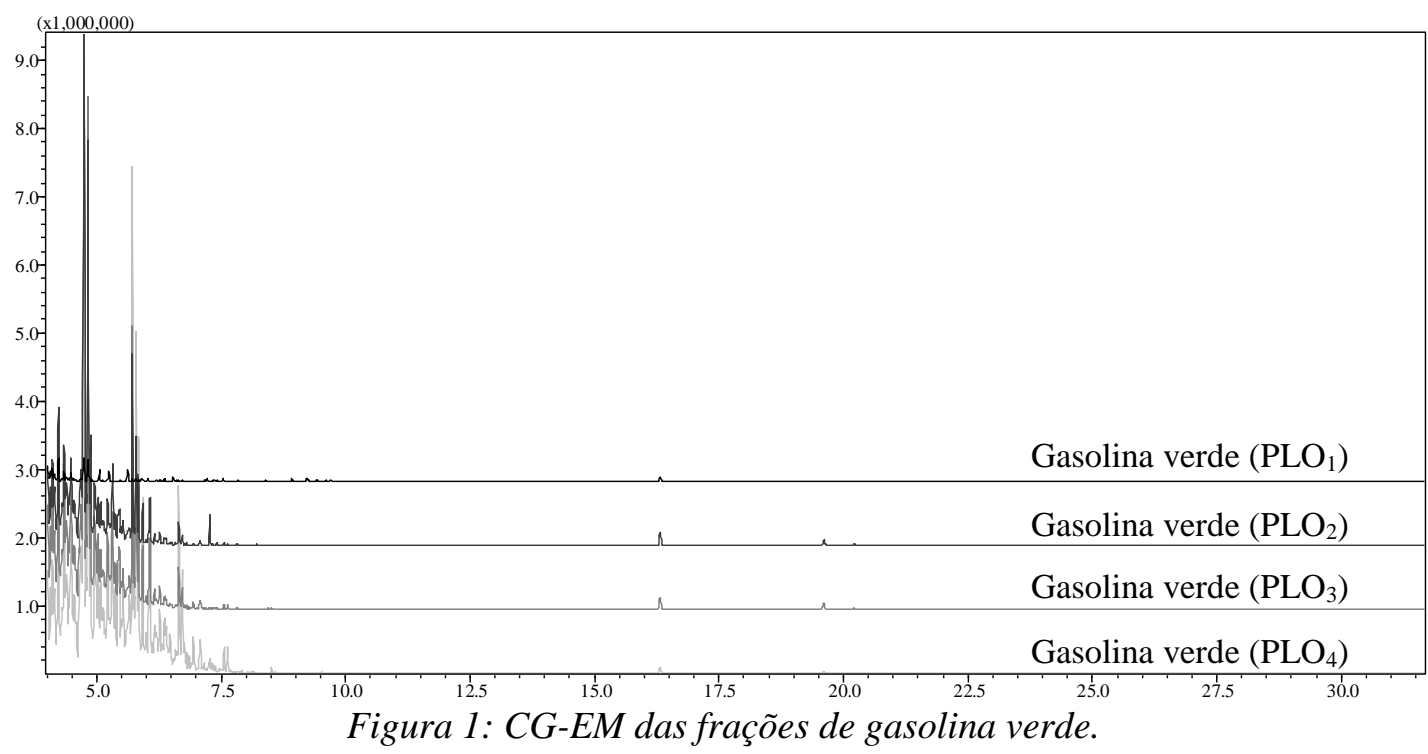

Os resultados apresentados na Tabela 4 mostram que à medida que há um incremento do conteúdo de catalisador no craqueamento térmico-catalítico de óleo de palma, o teor de hidrocarbonetos das frações de gasolina verde também aumenta, enquanto o teor de compostos oxigenados diminui. Em relação ao grupo de hidrocarbonetos, a classe mais representativa é a classe das olefinas, seguida das classes de naftênicos, parafinas normais e aromáticos, os quais seus teores variam de 16,25-42,31\%, 25,42-39,21\%, 14,70-21,525 e 0,00-2,57\%, respectivamente. Farah (2012) [20] e Szklo (2008) [21] relatam que os principais componentes presentes nas frações destiladas oriundas do petróleo são: parafinas normais, olefinas e naftênicos. Portanto, os resultados referentes às classes de hidrocarbonetos estão de acordo com aqueles apresentados na literatura. No que tange aos compostos de oxigenados, as cetonas constituem a classe com maior teor, seguido de álcoois, aldeídos e outros compostos.

De acordo com a Tabela 4, verificou-se que apenas o $\mathrm{PLO}_{1}$ produz frações de gasolina verde com hidrocarbonetos aromáticos e em pequenas concentrações. Portanto, no que tange a essa característica, as frações de gasolina verde devem ser melhoradas, pois segundo Farah (2012) [20], a presença de hidrocarbonetos aromáticos é uma das classes de hidrocarbonetos que influencia a resistência à detonação, uma vez que os aromáticos favorecem o poder antidetonante dos combustíveis utilizados nos motores de ignição por centelha como a gasolina. Em paralelo, Farah (2012) [20] também relata que a presença de parafinas normais implica uma maior estabilidade ou resistência à oxidação, promovendo a durabilidade do combustível. 
Tabela 3: Composição química das frações de gasolina verde.

\begin{tabular}{|c|c|c|c|c|c|}
\hline \multirow{2}{*}{ Compostos químicos } & \multirow{2}{*}{$\begin{array}{c}\text { Número } \\
\text { CAS }\end{array}$} & \multicolumn{4}{|c|}{ Gasolina verde } \\
\hline & & PLO $_{1}$ & $\mathrm{PLO}_{2}$ & $\mathrm{PLO}_{3}$ & $\mathrm{PLO}_{4}$ \\
\hline \multicolumn{6}{|l|}{ Área (\%) } \\
\hline Decano & $124-18-5$ & 14,70 & 0,00 & 0,00 & 12,13 \\
\hline Undecano & $1120-21-4$ & 0,00 & 21,16 & 19,85 & 7,26 \\
\hline Dodecano & $112-40-3$ & 0,00 & 0,00 & 0,00 & 1,67 \\
\hline Tetradecano & $629-59-4$ & 0,00 & 0,00 & 0,00 & 0,46 \\
\hline 4-Nonino & 20184-91-2 & 0,00 & 2,86 & 2,54 & 1,68 \\
\hline 1-Deceno & $872-5-9$ & 14,24 & 0,00 & 0,00 & 15,02 \\
\hline 4-Deceno & 19689-18-0 & 0,00 & 0,00 & 0,00 & 3,47 \\
\hline (E)-2-Deceno & $20063-97-2$ & 0,00 & 3,69 & 7,81 & 0,00 \\
\hline (Z)-2-Deceno & $20348-51-0$ & 0,00 & 3,31 & 0,00 & 4,12 \\
\hline 1,11-Dodecadieno & $5876-87-9$ & 0,00 & 0,00 & 1,33 & 0,00 \\
\hline 1-Dodeceno & $112-41-4$ & 0,00 & 24,74 & 27,65 & 12,78 \\
\hline (3E)-3-Dodeceno & $7239-23-8$ & 0,00 & 1,35 & 1,46 & 0,00 \\
\hline 1-Trideceno & $2437-56-1$ & 0,00 & 0,00 & 0,00 & 0,44 \\
\hline (3Z)-3-Tetradeceno & $41446-67-7$ & 2,01 & 0,00 & 0,00 & 0,00 \\
\hline 1-Hexadecino & $629-74-3$ & 0,00 & 0,00 & 1,52 & 0,00 \\
\hline Trans-1-Butenilciclopentano & $0-0-0$ & 6,05 & 0,00 & 0,00 & 0,00 \\
\hline 3-Butil-ciclohexeno & $3983-07-1$ & 0,00 & 0,00 & 0,00 & 1,00 \\
\hline Isobutilciclohexano & $3983-03-7$ & 14,98 & 5,38 & 5,32 & 3,75 \\
\hline 1-Butil-ciclopenteno & 2423-01-0 & 6,88 & 4,22 & 3,93 & 2,57 \\
\hline 3-Propil-ciclohexeno & $3983-06-0$ & 0,00 & 2,66 & 2,73 & 2,23 \\
\hline 1-Propil-ciclohexeno & $2539-75-5$ & 11,30 & 6,73 & 6,81 & 4,94 \\
\hline 3-Isobutil-1-ciclohexeno & $4104-56-7$ & 0,00 & 0,00 & 0,00 & 2,61 \\
\hline trans-1-Isopropenil-4-metilciclohexano & $124-25-0$ & 0,00 & 0,00 & 0,00 & 1,47 \\
\hline Butil-ciclohexano & $1678-93-9$ & 0,00 & 0,00 & 2,81 & 3,42 \\
\hline 2-Ciclohexilbutano & 7058-01-7 & 0,00 & 2,15 & 0,00 & 0,00 \\
\hline 1-Butil-ciclohexeno & $3282-53-9$ & 0,00 & 2,05 & 2,37 & 3,41 \\
\hline 1-Pentil-2-propil-ciclopropano & $41977-33-7$ & 0,00 & 2,23 & 2,25 & 4,58 \\
\hline Nonil-ciclopropano & $74663-85-7$ & 0,00 & 0,00 & 0,00 & 3,29 \\
\hline Propil-benzeno & $103-65-1$ & 2,57 & 0,00 & 0,00 & 0,00 \\
\hline Propanotriol & $56-81-5$ & 0,00 & 1,02 & 0,00 & 0,00 \\
\hline 2-Ciclohexiletanol & $4442-79-9$ & 0,00 & 1,67 & 2,01 & 0,00 \\
\hline 1-Dodecanol & $112-53-8$ & 0,00 & 6,85 & 1,13 & 0,00 \\
\hline 5-Norbornano-2-carboxaldeído & $5453-80-5$ & 7,09 & 0,00 & 0,00 & 0,00 \\
\hline Biciclo $[2,2,2]$ octano-2,3-diona & $4216-89-1$ & 0,00 & 1,79 & 1,73 & 2,12 \\
\hline 3-metil-Biciclo [3.3.0]oct-2-en-8-ona & $0-0-0$ & 20,18 & 0,00 & 0,00 & 0,00 \\
\hline Fenol & $108-95-2$ & 0,00 & 4,04 & 3,96 & 0,00 \\
\hline o-Cresol & $95-48-7$ & 0,00 & 2,10 & 2,14 & 5,58 \\
\hline p-Cresol & $106-44-5$ & 0,00 & 0,00 & 0,65 & 0,00 \\
\hline Total & & 100,00 & 100,00 & 100,00 & 100,00 \\
\hline
\end{tabular}


Tabela 4: Composição química das frações de gasolina verde em termos de hidrocarbonetos e compostos oxigenados.

\begin{tabular}{lcccc}
\hline \multirow{2}{*}{ Classe de compostos } & \multicolumn{4}{c}{ Gasolina verde } \\
\cline { 2 - 5 } & PLO $_{\mathbf{1}}$ & PLO $_{\mathbf{2}}$ & PLO $_{\mathbf{3}}$ & PLO $_{\mathbf{4}}$ \\
\hline Área (\%) & & & & \\
Hidrocarbonetos & 72,73 & 82,53 & 88,38 & 92,30 \\
Parafinas normais & 14,70 & 21,16 & 19,85 & 21,52 \\
Olefinas & 16,25 & 35,95 & 42,31 & 37,51 \\
Naftênicos & 39,21 & 25,42 & 26,22 & 33,27 \\
Aromáticos & 2,57 & 0,00 & 0,00 & 0,00 \\
Compostos oxigenados & 27,27 & 17,47 & 11,62 & 7,70 \\
Ácidos carboxílicos & 0,00 & 0,00 & 0,00 & 0,00 \\
Álcoois & 0,00 & 9,54 & 3,14 & 0,00 \\
Aldeídos & 7,09 & 0,00 & 0,00 & 0,00 \\
Cetonas & 20,18 & 1,79 & 1,73 & 2,12 \\
Outros & 0,00 & 6,14 & 6,75 & 5,58 \\
Total & 100,00 & 100,00 & 100,00 & 100,00 \\
\hline
\end{tabular}

Os resultados apresentados na Figura 2 mostram, primeiramente, que a destilação fracionada e o conteúdo de catalisador empregado para produzir os PLOs influenciaram significativamente a distribuição dos hidrocarbonetos no que tange ao número de carbonos que os constituem. Em segundo lugar, a Figura 2 mostra a presença de hidrocarbonetos de cadeia entre C9 e C16 nas frações de gasolina verde, as quais consistem principalmente de hidrocarbonetos com cadeias C9 a C12. O somatório dos teores de hidrocarbonetos C9 a C12 presentes nas frações de gasolina verde provenientes do fracionamento dos $\mathrm{PLO}_{1}, \mathrm{PLO}_{2}, \mathrm{PLO}_{3}$ e $\mathrm{PLO}_{4}$ totalizam 70,72\%, $82,53 \%, 86,86 \%$ e $91,40 \%$ em massa, respectivamente, atingindo quase que a totalidade dos teores em hidrocarbonetos em cada fração. De acordo com Speight (2006) [22], a gasolina derivada de petróleo é caracterizada por hidrocarbonetos com cadeias na faixa C4-C12. Portanto, o tamanho das cadeias de hidrocarbonetos que constituem as frações de gasolina verde obtidos no presente estudo está de acordo com a literatura.

Em relação aos resultados do presente trabalho, é importante destacar também que o $\mathrm{PLO}_{4}, \mathrm{o}$ qual é produto líquido orgânico produzido a partir do craqueamento térmico-catalítico de óleo de palma com $20 \%$ de catalisador, mostrou-se como a melhor matéria prima para obtenção da fração de gasolina verde. As razões pelas quais o $\mathrm{PLO}_{4}$ foi considerado a melhor matéria prima são: (1) o fracionamento do $\mathrm{PLO}_{4}$ promoveu o maior rendimento em fração de gasolina verde com 13,82\% em massa; (2) a fração de gasolina verde proveniente do fracionamento do $\mathrm{PLO}_{4}$ é a fração destilada que apresenta as melhores propriedades físico-químicas, principalmente, no que tange ao índice de acidez em função do seu menor valor obtido e (3) a fração de gasolina verde proveniente do fracionamento do $\mathrm{PLO}_{4}$ é a fração destilada que apresenta a melhor composição química, pois apresenta o maior teor de hidrocarbonetos, e consequentemente, o menor teor de compostos oxigenados, resultando em características favoráveis para um potencial substituto parcial e/ou total de derivados do petróleo. 


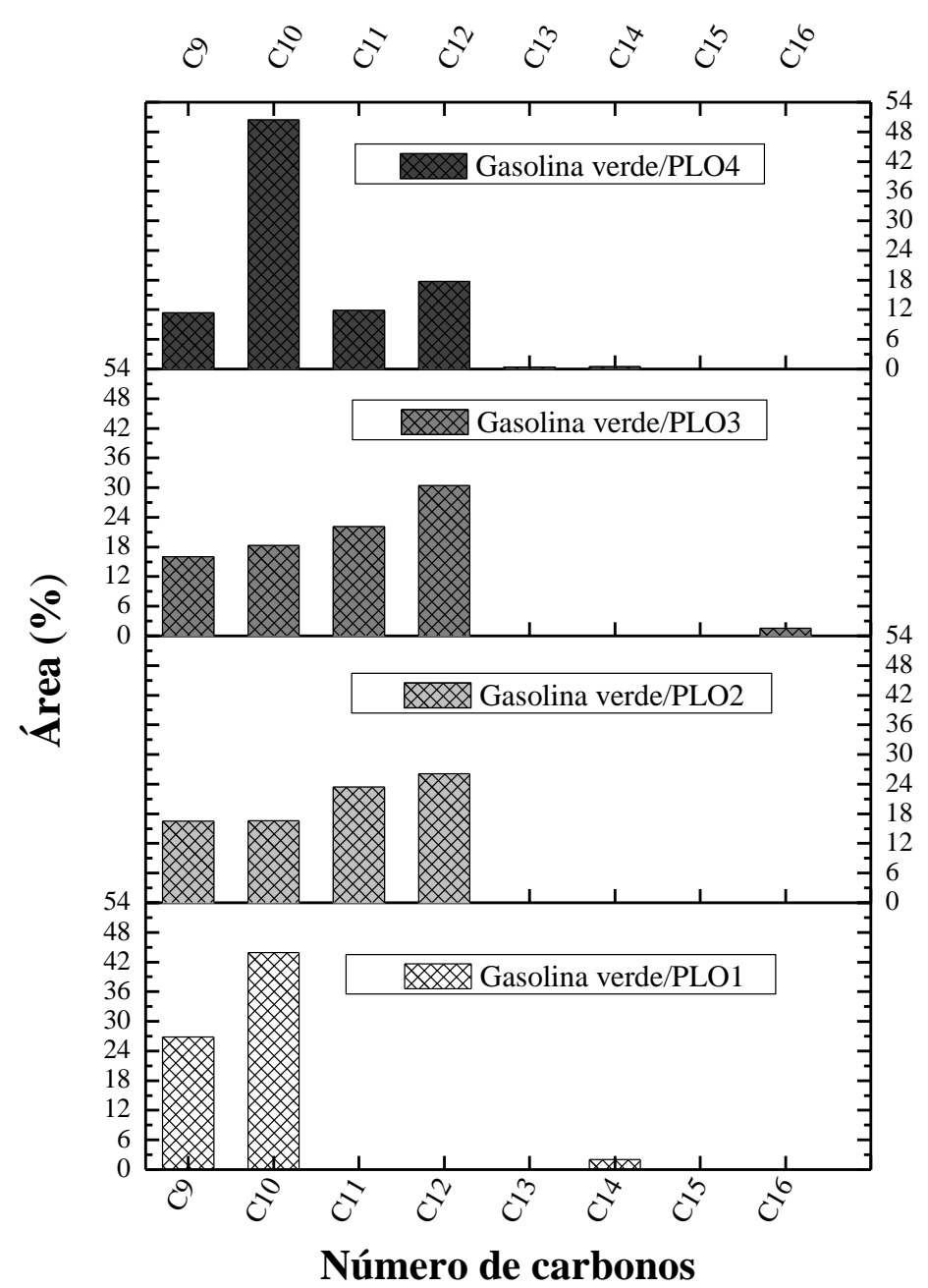

Figura 2: Distribuição do número de carbonos das frações de gasolina verde.

\section{CONCLUSÃO}

A partir dos resultados obtidos no presente trabalho, verificou-se que os valores de propriedades físico-químicas das frações de gasolina verde são inferiores àqueles valores dos seus respectivos PLOs, indicando que a destilação fracionada melhora as propriedades físicoquímicas das frações destiladas.

$\mathrm{O}$ incremento na porcentagem de catalisador no craqueamento térmico-catalítico para produzir o $\mathrm{PLO}_{1}, \mathrm{PLO}_{2}, \mathrm{PLO}_{3}$ e $\mathrm{PLO}_{4}$ : (1) promoveu um aumento gradual e significativo de 1,97 a $13,82 \%$ em massa nos valores de rendimento das frações de gasolina verde; (2) as propriedades físico-químicas como densidade, viscosidade e índice de refração das respectivas frações de gasolina verde também aumentaram, enquanto os valores de índice de acidez e índice de saponificação reduziram; (3) o teor de hidrocarbonetos das frações de gasolina verde também aumentou, enquanto o teor de compostos oxigenados diminuiu; (4) influenciou significativamente a distribuição dos hidrocarbonetos das frações de gasolina verde no que tange ao número de carbonos que os constituem, as quais consistem principalmente de hidrocarbonetos com cadeias $\mathrm{C} 9$ a $\mathrm{C} 12$.

O aumento nos valores de densidade, viscosidade e índice de refração é o resultado da presença de hidrocarbonetos com cadeias maiores à medida que ocorre a destilação fracionada do $\mathrm{PLO}_{1}$ ao $\mathrm{PLO}_{4}$. Em relação ao grupo de hidrocarbonetos, a classe mais representativa é a classe das olefinas, seguida das classes de naftênicos, parafinas normais e aromáticos. No que tange aos compostos de oxigenados, as cetonas constituem a classe com maior teor, seguido de 
álcoois, aldeídos e outros compostos. Por último, concluiu-se que o $\mathrm{PLO}_{4}$ é a melhor matéria prima para a obtenção da fração de gasolina verde, pois seu fracionamento promove o maior rendimento em massa, as melhores propriedades físico-químicas, bem como a melhor composição química entre as quatro frações de gasolina verde investigadas neste trabalho.

Portanto, as frações de gasolina verde podem ser utilizadas como um substituto parcial ou total da gasolina de petróleo e/ou como uma das frações que constituem o chamado "Pool" da gasolina de petróleo.

\section{AGRADECIMENTOS}

Este trabalho foi financiado pela Coordenação de Aperfeiçoamento de Pessoal de Nível Superior (CAPES) e Centrais Elétricas Brasileiras S/A. (Eletrobrás S/A).

\section{REFERÊNCIAS BIBLIOGRÁFICAS}

1. Dandik L, Aksoy HA, Erdem-Senatalar A. Catalytic Conversion of Used Oil to Hydrocarbon Fuels in a Fractionating Pyrolysis Reactor. Energy \& Fuels. 1998;12(6):1148-52, doi:10.1021/ef980012u.

2. Yan S, DiMaggio C, Wang H, Mohan S, Kim M, Yang L, et al. Catalytic Conversion of Triglycerides to Liquid Biofuels Through Transesterification, Cracking, and Hydrotreatment Processes. Current Catalysis. 2012;1(1):41-51, doi:10.2174/2211544711201010041.

3. Wang S. High-Efficiency Separation of Bio-Oil. 2013, doi:10.5772/51423.

4. Schwab AW, Dykstra GJ, Selke E, Sorenson SC, Pryde EH. Diesel fuel from thermal decomposition of soybean oil. Journal of the American Oil Chemists' Society. 1988;65(11):1781-6, doi:10.1007/BF02542382.

5. Prado CMR, Antoniosi Filho NR. Production and characterization of the biofuels obtained by thermal cracking and thermal catalytic cracking of vegetable oils. Journal of Analytical and Applied Pyrolysis. 2009;86(2):338-47, doi:10.1016/j.jaap.2009.08.005.

6. Twaiq FAA, Mohamad AR, Bhatia S. Performance of composite catalysts in palm oil cracking for the production of liquid fuels and chemicals. Fuel Processing Technology. 2004;85(11):1283-300, doi:10.1016/j.fuproc.2003.08.003.

7. Ooi Y-S, Zakaria R, Mohamed AR, Bhatia S. Synthesis of composite material MCM-41/Beta and its catalytic performance in waste used palm oil cracking. Applied Catalysis A: General. 2004;274(12):15-23, doi:10.1016/j.apcata.2004.05.011.

8. Wiggers VR, Meier HF, Wisniewski A, Barros AAC, Maciel MRW. Biofuels from continuous fast pyrolysis of soybean oil: A pilot plant study. Bioresource Technology. 2009;100(24):6570-7, doi:10.1016/j.biortech.2009.07.059.

9. Santos AL, Martins DU, Iha OK, Ribeiro RA, Quirino RL, Suarez PA. Agro-industrial residues as low-price feedstock for diesel-like fuel production by thermal cracking. Bioresour Technol. 2010;101(15):6157-62, doi:10.1016/j.biortech.2010.02.100.

10. Buzetzki E, Sidorová K, Cvengrošová Z, Kaszonyi A, Cvengroš J. The influence of zeolite catalysts on the products of rapeseed oil cracking. Fuel Processing Technology. 2011;92(8):1623-31, doi:10.1016/j.fuproc.2011.04.009.

11. Wiggers VR, Zonta GR, Franca AP, Scharf DR, Simionatto EL, Ender L, et al. Challenges associated with choosing operational conditions for triglyceride thermal cracking aiming to improve biofuel quality. Fuel. 2013;107:601-8, doi:10.1016/j.fuel.2012.11.011.

12. da Mota SAP, Mancio AA, Lhamas DEL, de Abreu DH, da Silva MS, dos Santos WG, et al. Production of green diesel by thermal catalytic cracking of crude palm oil (Elaeis guineensis Jacq) in a pilot plant. Journal of Analytical and Applied Pyrolysis. 2014;110:1-11, doi:10.1016/j.jaap.2014.06.011.

13. Mancio AA, da Costa KMB, Ferreira CC, Santos MC, Lhamas DEL, da Mota SAP, et al. Thermal catalytic cracking of crude palm oil at pilot scale: Effect of the percentage of $\mathrm{Na} 2 \mathrm{CO} 3$ on the quality of biofuels. Industrial Crops and Products. 2016;91:32-43, doi:10.1016/j.indcrop.2016.06.033.

14. Mrad N, Paraschiv M, Aloui F, Varuvel EG, Tazerout M, Nasrallah SB. Liquid hydrocarbon fuels from fish oil industrial residues by catalytic cracking. International Journal of Energy Research. 2013;37(9):1036-43, doi: 10.1002/er.2906.

15. Li H, Shen B, Kabalu JC, Nchare M. Enhancing the production of biofuels from cottonseed oil by fixed-fluidized bed catalytic cracking. Renewable Energy. 2009;34(4):1033-9, doi:10.1016/j.renene.2008.08.004. 
16. Riazi MR. Characterization and Properties of Petroleum Fractions. Pennsylvania: ASTM International; 2005.

17. Oasmaa A, Elliott DC, Korhonen J. Acidity of Biomass Fast Pyrolysis Bio-oils. Energy \& Fuels. 2010;24(12):6548-54, doi:10.1021/ef100935r.

18. ANP. No 40, 25.10.2013-DOU 28.10.2013.

19. Parkash S. Refining Processes Handbook. New York: Elsevier; 2003.

20. Farah MA. Petróleo e seus derivados: definição, constituição, aplicação, especificações, características de qualidade. Rio de Janeiro: LTC; 2012.

21. Szklo AS, Uller VC. Fundamentos do refino d petróleo: tecnologia e economia. Rio de Janeiro: Interciência; 2008.

22. Speight JG. The Chemistry and Technology of Petroleum. Fourth Edition ed. New York: CRC Press; 2006. 